\section{PA-090 UNDERSTANDING PATIENT DECISIONS TO TRANSFER OR DISENGAGE FROM HIV CARE AND TREATMENT IN ZAMBIA}

Stephanie Topp, ${ }^{1}$ Chanda Mwamba, ${ }^{2}$ Laura Beres, ${ }^{3}$ Nancy Padian, ${ }^{4}$

Izukanji Sikazwe, ${ }^{2}$ Elvin Geng, ${ }^{4}$ Charles Holmes ${ }^{3} .{ }^{1}$ James Cook University, Australia; ${ }^{2}$ CIDRZ, Zambia; ${ }^{3}$ Johns Hopkins University, United States of America; ${ }^{4}$ University of California San Francisco, United States of America

\subsection{6/bmjgh-2016-000260.122}

Background Despite widespread roll-out of free HIV care and treatment (C\&T), large numbers of HIV-positive Zambians are disengaged from care. Nested within a 4-province study of HIV C\&T outcomes, we explored how interactions between system hardware (tangible components) and system software (normative $\&$ behavioural components) at the service-delivery level influenced patients' decision to transfer or disengage from care.

Methods In-depth interviews were conducted with a stratified random sample of $75 \mathrm{HIV}$-infected adults from 4 provinces and five patient categories: currently in-care, pregnant in-care, disengaged, transferred (to another facility), friend/ family of deceased patient. Sixteen focus group discussions were convened with lay and professional healthcare workers (HCW) providers serving the same catchment areas. Audio transcripts were translated, transcribed and subject to deductive and inductive analysis guided by a modified social-ecological framework.

Results Health system 'hardware' factors influencing patient decisions included distance and chronic understaffing that resulted in long wait-times and administrative mistakes (e.g. loss of patient records). Health system 'software' factors included various aspects of clinic organisational culture. Examples are limited consideration of HCWs of the way employment or family circumstances affected patients' ability to adhere to protocol-driven treatment schedules and a harmful power dynamic that compelled patients to 'humble' themselves and 'obey' HCWs to avoid being 'punished'. Described by many as a problem of HCWs 'lacking heart' or 'having a bad attitude', these phenomena were often linked to experiences of disrespect and/or abuse that influenced decisions to transfer or leave C\&T. Conclusions Findings demonstrate a dynamic and compounding effect of health system 'hardware' and 'software' factors on decisions to transfer/disengage. Data suggest a need for: i) improvements in physical resourcing and structuring of HIV services; ii) a move away from exclusively static clinic-based service models and iii) revisions to policy enabling a re-orientation of pre-service training, clinic leadership and workplace incentives to encourage health-promoting, person-centred care. 